\title{
OUTCOME OF OMG URETHROPLASTY BY ASOPA, BARBAGLI AND KULKARNI TECHNIQUE FOR MANAGEMENT OF ANTERIOR URETHRAL STRICTURE : ARE RESULTS AFFECTED BY THE SURGICAL TECHNIQUE?
}

MD. SHARIFUL ISLAM ${ }^{1}$, MD. ASADUZZAMMAN ${ }^{1}$, MD. RUHUL QUDDUS ${ }^{2}$, WALIUL ISLAM ${ }^{1}$, PRANASHIS SAHA $^{1}$

${ }^{1}$ Department of urology, National Institute of Kidney Diseases \& Urology, ${ }^{2}$ Satkhira Medical College, Satkhira

\begin{abstract}
Objective: To determine outcome of OMG urethroplasty by different surgical technique comparing ASOPA, BARBAGLI and KULKARNI TECHNIQUE in the management of anterior urethral stricture.

Materials and Methods: A total of 62 patients with an average age of 42 yr(21-55) underwent OMG urethroplasty between 2008 to 2015 for anterior urethral stricture of different etiology. 2 patient lost durig follow up patient with lichen sclerosus and failed hypospadias were not included. OMG was always harvested from cheek using 2 team approach graft were placed using surgical technique of ASOPA, BARBAGLI and KULKARNI technique in 21,27 and 12 patients respectively clinical outcome was considered success or failure at the time any post operative procedure needed mean follow up was 45 month (6-95)

Results: Out of 60 cases 51(85\%) considered success and 9(15\%) were considered failure. success rate were $85.7 \%, 85 \%$, and $83.5 \%$ in ASOPA, BARBAGLI and KULKARNI Technique and failure rate were $14.3 \%, 15 \%$ and $16.7 \%$ respectively. Failure involve in the anastomotic site ( 2 in distal and 3 in proximal) were managed by OIU and involving whole length in 3 patient managed with staged urethropllasty.

Conclusion: In our series, success rate of ASOPA, BARBAGLI and KULKARNI technique has no statistically significant variation. more over stricture recurrence was uniformly distributed in all group. so the outcome is not significantly affected by different technique used in OMG urethroplasty for anterior urethral stricture.
\end{abstract}

Key words Urethra, Urethral stricture, Oral mucosa, Urethroplasty

Bangladesh J. Urol. 2016; 19(1): 23-27

\section{Introduction}

Urethral stricture is one of the oldest problem faced by the urologist occurs due to fibrotic narrowing of urethral lumen and fibrosis usually extend into the surrounding corpus spongiosum causing spongiofibrosis. The gold standard treatment of urethral stricture is urethroplasty[1]. Buccal mucosa is architecturally similar to stratified squamous epithelium of penile and glanular urethra making it exceptionally adaptable for urethral

Address of Correspondence: Md. Shariful Islam, Department of Urology, National Institute of Kidney Diseases \& Urology, Email: drmsislam9@gmail.com reconstruction[2]. Whether to place the graft dorsally, ventrally or laterally is still controversial now. Single ventral graft is more prone to fistula formation, sacculation and diverticula formation leading to urinary stasis and ejaculatory dysfunction[3]. On the other hand, dorsal onlay graft procedure provides the advantages of better mechanical support by the corporal bodies with fewer incidences of sacculation and fistula formation but this approach may damage erectile function and the bulbar arteries when the dissection from the corpora needs to be very proximal[1-8], Asopa describe a technique of dorsal free graft by ventral sagittal 
urethrotomy approach without mobilizing the urethra there by vascularity of the urethra is not hampered. Kulkarni et al. describe one sided anterior urethroplasty - a new dorsal onlay graft technique for repair of anterior urethral stricture to preserve vascular supply to the urethra and its entire muscular and neurogenic support. We performed a retrospective outcome analysis of 60 patients who underwent 3 types of urethroplasty with the buccal mucosal graft-by Asopa, Barbagli and Kulkarni technique.

\section{Patients and Methods}

A total of 62 patients with an average age of 42 years (range 21 to 55) underwent buccal mucosa graft urethroplasty between January 2008 and september 2015 for bulbar urethral strictures. Patients with lichen sclerosus, failed hypospadias repair, previous failed open urethroplasty were not included in this study. The grafts were placed on dorsal onlay (Barbagli procedure), the ventral sagital urethrotomy with inlay patch (Asopa technique) and dorso-lateral onlay by unilateral mobilization of urethra (Kulkarni technique) in 27, 21 and 12 patients, respectively. The surgical technique was selected according to the site and length of the stricture within the bulbar urethra. All surgical procedures were performed in (NIKDU) and other specialized hospital in Dhaka by different urologists. There were no significant differences in the 3 groups in respect to mean patient age ,mean stricture length, etiology or mean graft length.

Preoperative evaluation included clinical history, physical examination, urine culture, residual urine measurement, uroflometry, and retrograde urethrogram and urethrocystoscopy. The etiology of stricture was ischemia in 18 cases, trauma in 10, instrumentation in 6 and unknown in 28. Average stricture length was $4 \mathrm{~cm}$ (range 2.5 to 7.5 ). The urethral procedure was done with the patient in the normal lithotomy position.

In 21 patients with strictures located in the penile and distal bulbar urethra. a longitudinal, ventral urethrotomy was made to open the strictured tract and dorsal midline urethrotomy incision extending $2 \mathrm{~cm}$ into normal urethra is done to place graft. The buccal mucosa graft was harvested from the cheek using a 2 team approach with the donor site closed with 4-zero polyglactin interrupted stitches. The buccal mucosa graft was defatted and tailored according to the length of the urethral opening by limiting graft width to $2.5 \mathrm{~cm}$. Mean graft length was $4.2 \mathrm{~cm}$. Three interrupted sutures were placed at the apexes of the distal and proximal anastomosis to avoid narrowing at these precarious sites. The graft was then sutured laterally to the left side of the urethral mucosa in running fashion using 6-zero polyglactin. The graft was then sutured laterally to the right side of the urethral mucosa in running fashion. A 14Fr silicone Foley catheter was inserted at this point. Ventral urethrotomy is closed with 5-zero polyglactin suture in running fashion. Spongiosum tissue was closed over the graft using 4zero polyglactin interrupted stitches

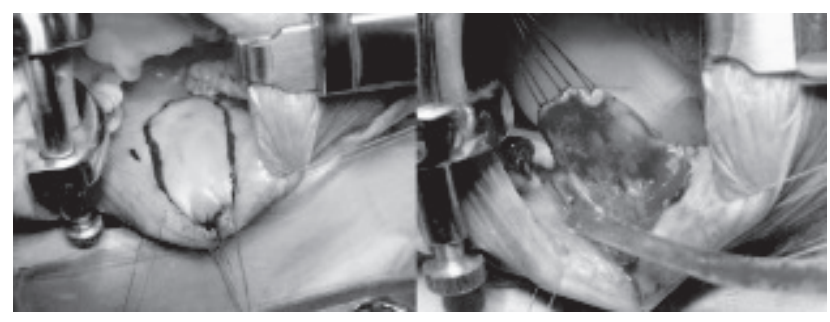

Fig.1: Harvesting of OMG

In 27 patients with strictures located in the distal part of the bulbar urethra the urethra was dissected from the corpora cavernosa and rotated 180 degrees. The dorsal urethral surface was exposed and fully opened. The distal and proximal lumina were calibrated. The buccal mucosa graft was sutured, splayed and quilted over the corpora cavernosa using 5-zero polyglactin interrupted stitches without limiting graft width $(3.5 \mathrm{~cm})$. The left side of the urethral mucosa was sutured to the graft using 5-zero polyglactin interrupted stitches. A $14 \mathrm{Fr}$ silicone Foley catheter was inserted at this point. The urethra was rotated back to its original position and sutured laterally to the right side of the graft. At the end of the procedure the graft was completely covered by the urethra.

In 12 patients with a full-sized urethral bulb the ventral urethral opening may create serious bleeding from particularly thick and highly vascular spongy tissue, and the dorsal approach to the urethra may damage erectile function when dissecting the urethra from the corpora cavernosa more proximally.In these patients the urethra was mobilized from midline on the ventral aspect to beyond the midline on the dorsal aspect. The urethra was opened in the mid line dorsally. Proximally, the graft was sutured to the open urethra by a few interrupted sutures at the apex and the medial urethral margin. Then one edge of the graft was sutured to the medial urethral margin by continuous suturing using $5 / 0$ vicryl until distal apex where few interrupted sutures given. A $14 \mathrm{Fr}$ folley catheter was kept. The other margin of the graft was sutured to the lateral margin of urethra and the tunica of corpora taking care to prevent stretching of graft. A corrugated drain was kept and wound closed in layers. 

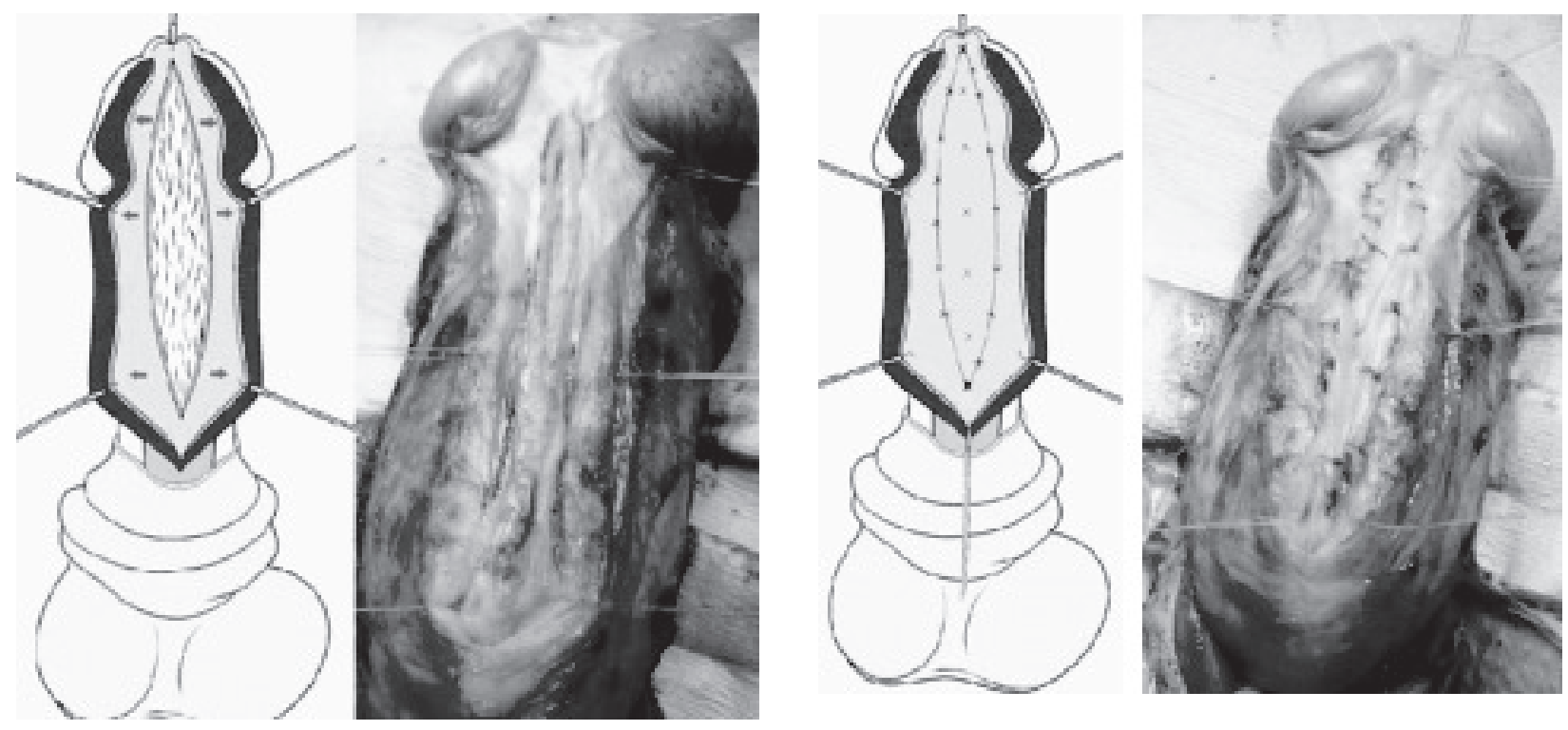

Fig.-2: OMG With ASOPA technique
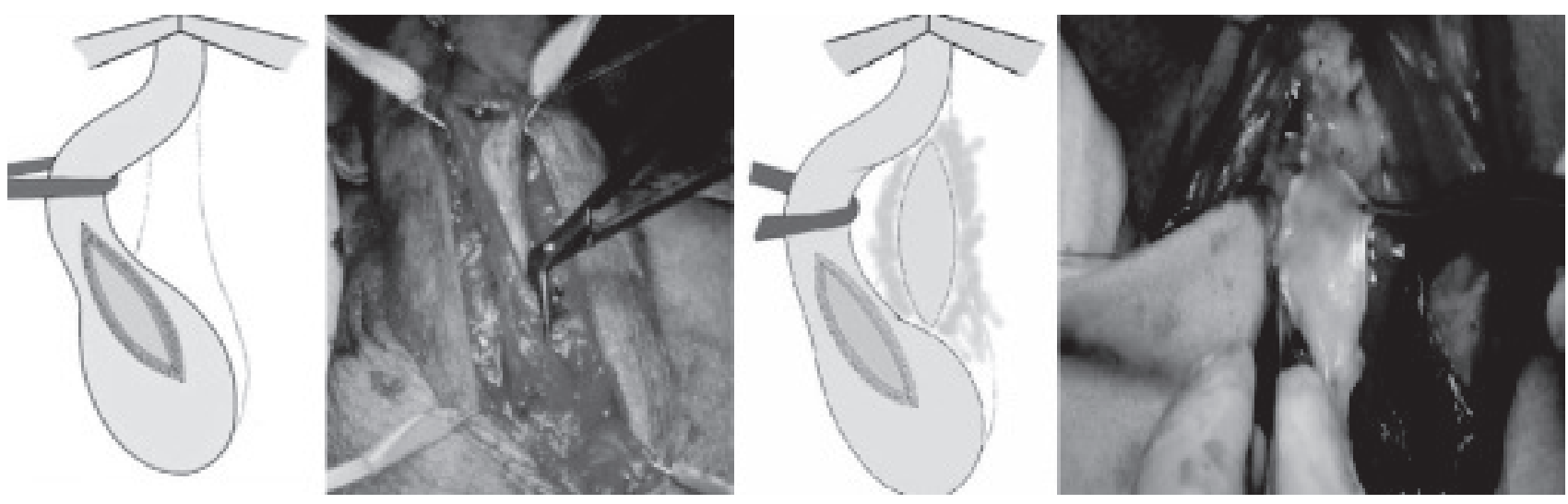

Fig.-3: OMG with BARBAGLI technique
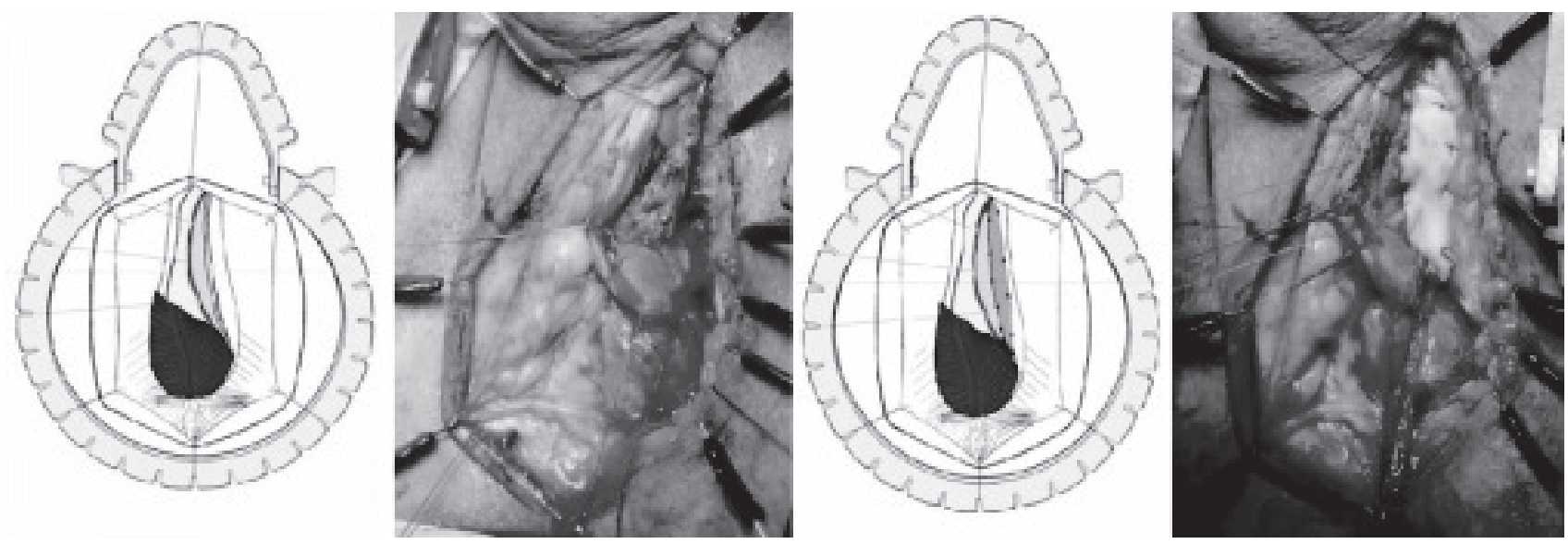

Fig.-4: OMG BY KULKARNI technique 
Postoperative management Patients were discharged home 3 days after surgery. Voiding cystourethrography was performed when the catheter was removed 3 weeks after surgery. If contrast extravasation was present, a $12 \mathrm{Fr}$ Foley catheter was reinserted and maintained for an additional 2 weeks. The clinical outcome was considered a success or a failure at the time that any postoperative procedure was needed, including dilation. Uroflowmetry and urine cultures were repeated every 4 months in year 1 and annually thereafter. When symptoms of decreased force of stream were present and uroflowmetry was less than $14 \mathrm{ml}$ per second, retrograde and voiding urethrography, and urethroscopy were repeated. Average followup was 45 months (range 06 to 93). A total of 37 patients with follow up longer than 48 months were clinically investigated to detect post-void dribbling and semen sequestration after ejaculation.

\section{Results}

Of 60 cases $51(85 \%)$ were considered success and 9 (15\%) were considered failures. In 21 patient of Asopa technique success were in 18 cases $(85.7 \%)$ and failure in $3(14.3 \%)$. The 27 dorsal graft (barbagli) provided success in 23 cases (85\%) and failure in 4 (15\%). The 12 lateral grafts (kulkarni) provided success in 10 cases $(83.3 \%)$ and failure in $2(16.7 \%)$. There were no early postoperative complications, such as wound infections, hematomas, bleeding requiring transfusion or neuronal injuries. No fistulas were evident at the first radiological investigation. In patients with stricture recurrence the endoscopic evaluation provided useful information about disease extension. The 9 failures involved the anastomotic site in 5 patients, including 2 in the ASOPA, 2 in the BARBAGLI and 1 in the KULKARNI group, and the whole grafted area in 4, including 2 in the BARBAGLI and 1 in the ASOPAgroup and 1 in the KULKARNI group. Four patients with restructure involving the entire grafted area were initially treated with perineal urethrostomy. Subsequently all patients underwent successful perineal urethrostomy closure using a new buccal mucosa graft according to our original technique. Two failures occurred at the distal graft Anastomosis and 3 occurred at the proximal graft anastomosis. These 5 patients were successfully treated with internal urethrotomy. 5 OF 37 PATIENT with follow up more than 48 month showed clinical symptom due to urethral sacculation. 2 each in barbagli and ASOPA and 1 in kulkarni. No patients required surgery for urethrocele correction.

\section{Discussion}

The use of buccal mucosa in bulbar urethroplasty should be modulated according to the exact stricture location and its characteristics. Placement of the graft on the dorsal urethral surface is simpler and safer in the distal part of the bulbar urethra, whereas ventral urethrotomy and dorsal inlay placement of the graft is more efficacious in the proximal part of the bulbar urethra, where the spongiosum tissue is thicker and better vascularized. Finally, in patients with long urethral strictures located in a full-sized urethral bulb, the lateral opening of the urethral surface can avoid the pitfall of ventral or dorsal urethrotomy.

In our series of patients recurrent stricture was uniformly distributed in Asopa, Barbagli and Kulkarni technique, occurring in 5 patients $(8.33 \%)$ as a white fibrous ring at the distal or proximal anastomotic sites. Other groups have found these rings, causing stricture recurrence[5,7]. Guralnick and Webster reported 2 recurrent strictures at the proximal and distal limit of repair in 29 patients who underwent augmented anastomotic urethroplasty, which were successfully treated with dilation and optical urethrotomy[9]. Graft failure at the distal anastomosis, where the corpus spongiosum is less vascular, may represent poor inosculation due to poor graft bed vascularity[7]. On the contrary, graft failure at the proximal anastomosis is currently attributed to under staging disease during surgery[7]. Moreover, the proximal anastomosis is deep in the bulb, which is a more difficult site technically in which to ensure epithelium-to-urothelium placement ${ }^{7}$. Further study is necessary to clarify the true etiology of this kind of repeat structuring. Unfortunately graft failure at the anastomotic sites significantly contributes to the deterioration of the final outcome of these bulbar urethroplasties. In the current series of patients, excluding failures due to anastomotic rings, the success rate increased from $85 \%$ to $93.3 \%$. Elliot et al reported a $90 \%$ success rate after bulbar buccal mucosa grafts[7]. Bulbar Urethroplasty Using Buccal Mucosa Grafts in a study of 957 patients by Gudio Barbagli suggesting that this rate can be improved (97\%) with internal urethrotomy for the management of anastomotic ring failures[10].

Urethrocele with post-void dribbling and semen sequestration are common problems after any kind of unsupported substitution urethroplasty[2,4,8,9]. In our series we observed clinical symptoms due to urethrocele in 8 patients but radiological evidence of urethral weakening was evident in only 6 . Mechanical urethral 
weakening was clinically detected in patients with follow up longer than 24 months, with a graft length of more than $4.5 \mathrm{~cm}$ and on voiding cystourethrography because retrograde urethrography failed to show urethral sacculation. Moreover, we also observed the clinical presence or post-void dribbling in 2 patients in whom voiding cystourethrography was normal. Recently Yucel and Baskin reported that perineal nerves innervate the bulbospongiosus muscles and send fine branches to penetrate the corpus spongiosum, mainly in the bulbar area, by piercing the junction of 2 bulbospongiosus muscles in the midline[11]. Rhythmic contractions of the bulbospongiosus muscles and other perineal muscles expel semen from the urethra and probably have an important role in expelling urine, avoiding urine sequestration in the large urethral bulb. Further studies are necessary to clarify if during bulbar urethroplasty surgical damage to the branches of the perineal nerves may have a role in determining a loss of efficient bulbar urethral contraction, causing difficulties in expelling semen and urine, and temporary or permanent sexual dysfunction[12].

\section{Conclusion}

It is important to remember despite meticulous technique all urehroplasty procedure have potential to fail or detoriate with time, so life long follow up is needed. The different technique of the graft placement showed no difference in the success rate. Currently the best technique is probably the one with which the urological reconstructive surgeon is most comfortable. No single technique is appropriate in all situations and the successful surgeon will have a repertoire of methods from which to choose. Further studies are necessary to clarify the etiology of disease recurrence at the apexes of the proximal and/or distal anastomoses between the urethra and grafts to improve our success rate.

\section{Conflict of Interest : None declared}

\section{References}

1. Armenakas, N.A.: Long-term outcome of ventral buccal mucosal grafts for anterior urethral strictures. AUA News, 9: 17, 2014.
2. Andrich, D. E., Leach, C. J. and Mundy A. R.: The Barbagli procedure gives the best results for patch urethroplasty of the bulbar urethra. BJU Int, 88: 385, 2001.

3. Barbagli, G., Selli, C., Tosto, A. and Palminteri, E.: Dorsal free graft urethroplasty. J Urol, 155: 123, 1996.

4. Iselin, C. E. and Webster, G. D.: Dorsal onlay graft urethroplasty for repair of bulbar urethral stricture. J Urol, 161: 815, 2009.

5. Barbagli, G., Palminteri, E. and Rizzo, M.: Dorsal onlay graft urethroplasty using penile skin or buccal mucosa in adult bulbourethral strictures. J Urol, 160: 1307, 1998.

6. Kane, C. J., Tarman, G. J., Summerton, D. J., Buchmann, C. E.,Ward, J. F., O'Reilly, K. J. et al: Multi-institutional experience with buccal mucosa onlay urethroplasty for bulbar urethral reconstruction. J Urol, 267: 1314, 2012.

7. Elliot, S. P., Metro, M. J. and McAninch, J. W.: Long-term followup of the ventrally placed buccal mucosa onlay graft in bulbar urethral reconstruction. J Urol, 169: 1754, 2003.

8. Bhandari, M., Dubey, D. and Verma, B. S.: Dorsal or ventral placement of the preputial/penile skin onlay flap for anterior urethral strictures: does it make a difference? BJU Int, 88: 39, 2001.

9. Dubey D., Kumar, A., Bansal, P., Srivastava, A., Kapoor, R.,Mandhani, A. et al: Substitution urethroplasty for anterior urethral strictures: a critical appraisal of various techniques. BJU Int, 91: 215, 2003.

10. Palminteri, E., Lazzeri, M., Guazzoni, G., Turini, D. and Barbagli, G.: New 2-stage buccal mucosal graft urethroplasty. J Urol 167: 130, 2002.

13. Yucel, S. and Baskin, L. S.: Neuroanatomy of the male urethra and perineum. BJU Int, 92: 624, 2003.

14. Andrich DE, Dunglison N, Greenwell TJ. and Mundy AR. The long-term results of urethroplasty. J Urol, 170: 90, 2003.

Abbreviations:

OMG : Oral Mucosal Graft 